\title{
Oxidized low-density lipoprotein as a biomarker of in vivo oxidative stress: from atherosclerosis to periodontitis
}

\author{
Hiroyuki Itabe* \\ Department of Biological Chemistry, Showa University School of Pharmacy, 1-5-8 Hatanodai, Shinagawa-ku, Tokyo 142-8555, Japan
}

(Received 14 November, 2011; Accepted 28 November, 2011; Published online 30 March, 2012)

\begin{abstract}
Oxidized low-density lipoprotein is known as an important factor in the development of atherosclerosis. The introduction of a sensitive procedure for the determination of oxidized low-density lipoprotein in human circulating plasma using a monoclonal antibody recognizing oxidized phosphatidylcholines has opened new fields of research based on in vivo oxidized low-density lipoprotein. The plasma oxidized low-density lipoprotein levels are significantly elevated in patients with acute myocardial infarction, cerebral infarction or chronic renal failure accompanied by hemodialysis. It was found that the plasma oxidized low-density lipoprotein level increased prior to aortic atherosclerotic lesion enlargement in apolipoprotein E-knockout mice. Recent studies have pointed out that oxidized low-density lipoprotein is transferrable between vessel wall tissue and the circulation, so it is a reasonable hypothesis that plasma oxidized low-density lipoprotein levels reflect the oxidative status at local sites of atherogenesis. Oxidized low-density lipoprotein measurement has been applied to human gingival crevicular fluids, which can be collected easily and safely, and relatively high levels of oxidized lowdensity lipoprotein were shown to be present. These findings, together with recent clinical follow-up studies, suggest that oxidized low-density lipoprotein is a predictive biomarker of a variety of diseases related to oxidative stress. This review summarizes the current understanding of in vivo oxidized lowdensity lipoprotein and its potential significance as a biomarker of disease.
\end{abstract}

\section{Key Words: oxidized low-density lipoprotein, oxidized phophatidylcholine, oxidative stress, atherosclerosis, periodontitis}

\section{Detection of OxLDL in Atherosclerotic Lesions}

Oxidative stress is involved in a variety of pathological conditions, such as inflammation, atherosclerosis, obesity, and cancer. Aerobic organisms cannot live without oxygen, and oxygen is an active molecule which elicits oxidative modifications of essential compounds of our body. In particular, polyunsaturated fatty acids (PUFA) are molecules very susceptible to oxidation reactions.

Low-density lipoprotein (LDL) is a major carrier of cholesterol in the circulation. Since cholesterol is insoluble in water, it resides in a large complex composed of various lipids and proteins, which is called lipoprotein. Triglycerides and cholesteryl esters are packed in the core of the lipoprotein particles, and it is surrounded by a phospholipid monolayer sheet. In each LDL particle, one molecule of apolipoprotein B-100 (apoB) is included (Fig. 1). Nearly $80 \%$ of human LDL is composed of lipids, and more than a half of the lipid molecules are PUFA-containing lipids. ${ }^{(1)}$ When LDL is exposed to oxidation, the PUFA moieties in those lipids are easily oxidized so as to produce a variety of oxidation products containing oxidized functional groups, such as hydroperoxides, epoxides, endoperoxides, isoprostanes, aldehydes, carboxylic acids and unsaturated ketones. They are chemically active and induce secondary reactions with the side chains of amino acid residues in apolipoproteins. Oxidized low-density lipoprotein (OxLDL) is characterized by the presence of various oxidized lipids and amino acid residues modified with oxidized lipids, and OxLDL is thus a mixture of those heterogeneously modified LDL particles.

Foam cells, which accumulate neutral lipids in cytoplasmic lipid droplets, are a typical feature of atherosclerotic lesions. Chemically modified forms of LDL were first reported to induce foam cell formation by macrophages in the 1980s..$^{(2)}$ In particular, the oxidation of LDL was found to occur under various physiological and phathological conditions. This suggested that lipid oxidation and its products could be important clues to understanding the molecular basis of a variety of diseases. Subsequently, macrophage scavenger receptors, which recognize chemically modified LDL, including OxLDL, came to be extensively studied. The first scavenger receptor identified and cloned was SR-A.(3) Since then, a number of scavenger receptors have been identified. ${ }^{(4)}$ These in vitro studies strengthened the OxLDL hypothesis, but further studies are still needed to obtain corroborating in vivo evidence for the variety of activities of OxLDL.

We have tried to identify the specific materials which are present in atherosclerotic lesions by raising lesion-related monoclonal antibodies (mAb) ${ }^{(5-7)}$ Among many antibodies against atherosclerotic lesions, a clone that strongly reacts with copper-induced OxLDL was raised. ${ }^{(8)}$ The antibody, DLH3, recognizes oxidized phosphatidylcholines (OxPC), where 1palmitoyl-2-(9-oxo)nonanoyl-PC (9CHO-PC, also called PONPC) is one of the potent antigenic molecules (Fig. 1). ${ }^{(9)}$

It has been extensively studied that various short-chain containing PC molecules are produced during oxidative modification. ${ }^{(10)}$ Fig. 1B shows a presumed scheme of 9CHO-PC formation. Linoleate moiety in the PC molecule will be attacked at 9- or 13-position of the acyl chain. Then 9-alkoxy radical could cleave $\mathrm{C}-\mathrm{C}$ bond at 9-10 position to form 9-carbon aldehydecontaining PC.

The antibody DLH3 was also shown to be useful immunohistochemically as a probe for the detection of OxPC in combination with an anti-apoB polyclonal antibody (pAb). In human atherosclerotic lesions, immuno-double staining or adjacent serial

\footnotetext{
*To whom correspondence should be addressed.

E-mail: h-itabe@pharm.showa-u.ac.jp
}

He received "SFRR Japan Award of Scientific Excellent" in 2011 in recognition of his outstanding work. 
A

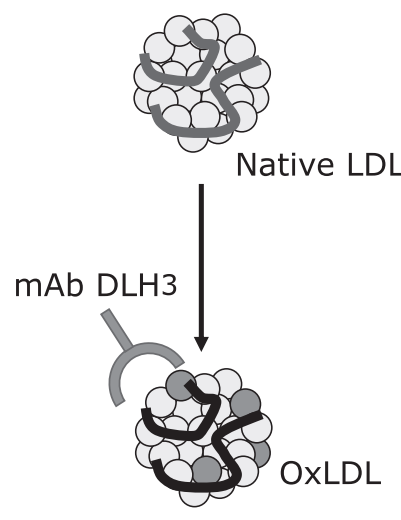

OxPC
B

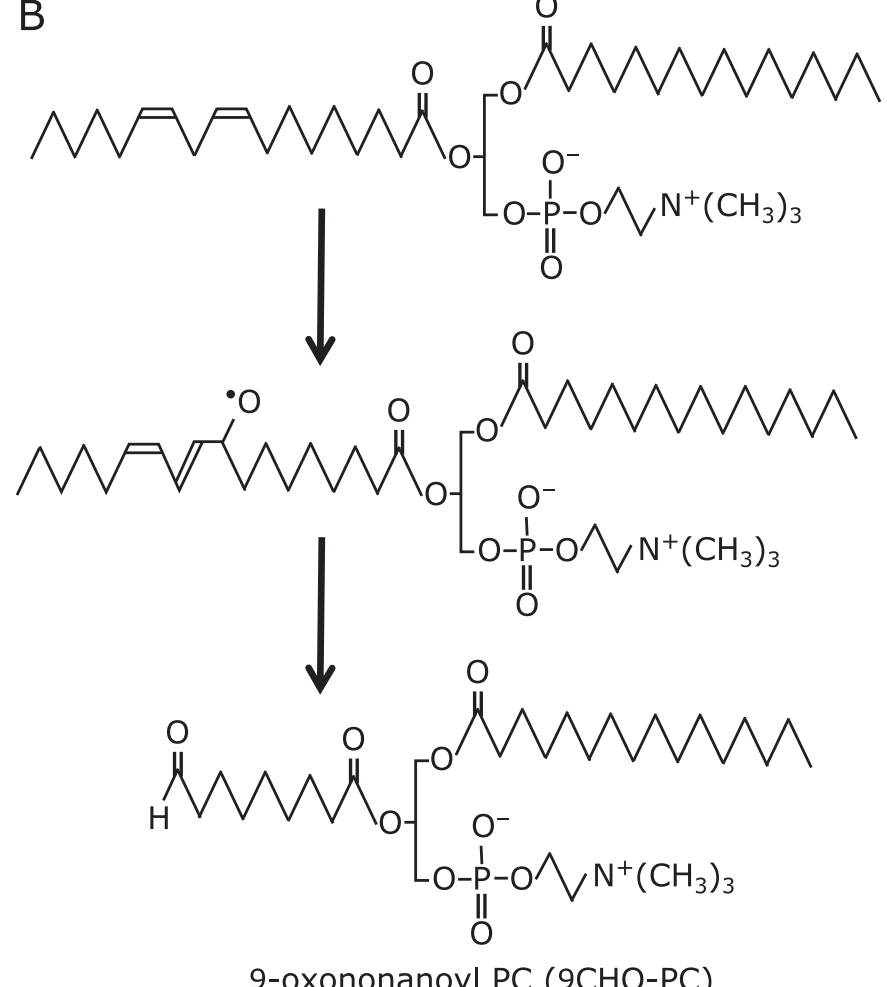

Fig. 1. Anti-OxLDL mAb DLH3 recognizes OxPC. A: LDL is composed of a number of lipid molecules surrounded by a single molecule of apolipoprotein B-100 (apoB). When LDL is oxidized, lipid peroxidation products are primarily derived from PUFA-containing phospholipids and triacylglycerol, and some of the peroxidation products bind to $\mathrm{apoB}$, thereby modifying the protein structures. B: One of the lipid oxidation products containing a $\mathrm{PC}$ backbone and an aldehyde group, 9CHO-PC. This oxidized PC is recognized by the anti-OxPC mAb DLH3. It is very likely that the 1-palmitoyl-2linoleoyl PC is the precursor of 9CHO-PC. Linoleate moiety in the PC molecule will be attacked at 9- or 13-position of the acyl chain. Then 9-alkoxy radical could cleave C-C bond at9-10 position to form 9-carbon aldehyde-containing PC.

section staining allowed the observation that OxPC was often specifically present together with apoB in macrophage-derived foam cells. ${ }^{(8,11,12)}$ The presence of OxLDL in human atherosclerotic lesions was further demonstrated by analysis of the lipoprotein fraction extracted from carotid plaques obtained from endarterectomized patients. ${ }^{(12)}$ When either OxLDL or acetylated LDL is incubated with macrophages in culture, the macrophages take up the modified LDL particles and then degrade them in their lysosomes. But the hydrolysis of the modified apoB in the OxLDL was reported to be incomplete compared with acetylated LDL in macrophage lysosomes. ${ }^{(13,14)}$ Interestingly, the OxLDL extracted from the carotid artery lesions was partially degraded. ${ }^{(12)}$ It ranges between $50-150 \mathrm{kDa}$, while the native apoB-100 is approximately $500 \mathrm{kDa}$, suggesting that the OxLDL in the lesions is processed by the macrophages. This observation is a good evidence for the presence of OxLDL in vivo (Fig. 2).

\section{Circulating OxLDL and Cardiovascular Diseases}

The anti-OxPC antibody was utilized to establish a sandwich enzyme-linked immunosorbent assay (ELISA) procedure for detecting even small amounts of OxLDL in human plasma. ${ }^{(15)}$ The OxLDL present in human plasma LDL fraction was shown to be detectable even in the LDL from healthy subjects, even though this amount of OxLDL (approximately $0.1 \mathrm{ng} / \mu \mathrm{g}$ LDL protein) was very small. The OxLDL levels of LDL fraction from patients with acute myocardial infarction (AMI), cerebral infarction or chronic renal failure were significantly higher than control subjects. ${ }^{(8,11,15,16)}$
Several research groups have reported methods for the determination of OxLDL in human plasma with mAbs. These methods use similar but different $\mathrm{mAbs}$, as well as different ELISA strategies and standard materials. Thus, the OxLDL values obtained by these methods cannot be directly compared. Despite such formal technical differences in these procedures, the changes in OxLDL levels in patients with severe cardiovascular diseases, including AMI, were detected by all of these methods, which provide strong evidence that the circulating OxLDL level changes under these pathological conditions. ${ }^{(17)}$

Sandwich ELISA using a DLH3 antibody and anti-apoB pAb has been shown to be a powerful tool for studying the changes in the OxLDL levels in human plasma. The plasma OxLDL level is significantly increased in patients with AMI compared with normal subjects. ${ }^{(11)}$ In our procedure, the LDL fraction was separated from each plasma sample using ultracentrifugation, therefore the value obtained by the measurement is indicated as "ng of OxLDL per $\mu \mathrm{g}$ of LDL protein", which represents the degree of oxidation in the LDL particles.

The time course of the change in the plasma OxLDL levels in patients with AMI during the time of treatment in the hospital was studied. It was demonstrated that the plasma OxLDL levels were significantly high in the acute phase after administration in the hospital, but went down to a nearly normal level by the time of discharge. ${ }^{(18)}$ A similar temporal change in plasma OxLDL was observed in the patients with cerebral infarction. ${ }^{(16)}$ These findings suggest that plasma OxLDL is released into the circulation from atherosclerotic lesions upon plaque rapture (Fig. 3). ${ }^{(19)}$

Tsimikas et al. ${ }^{(20)}$ have extensively studied the plasma OxLDL 


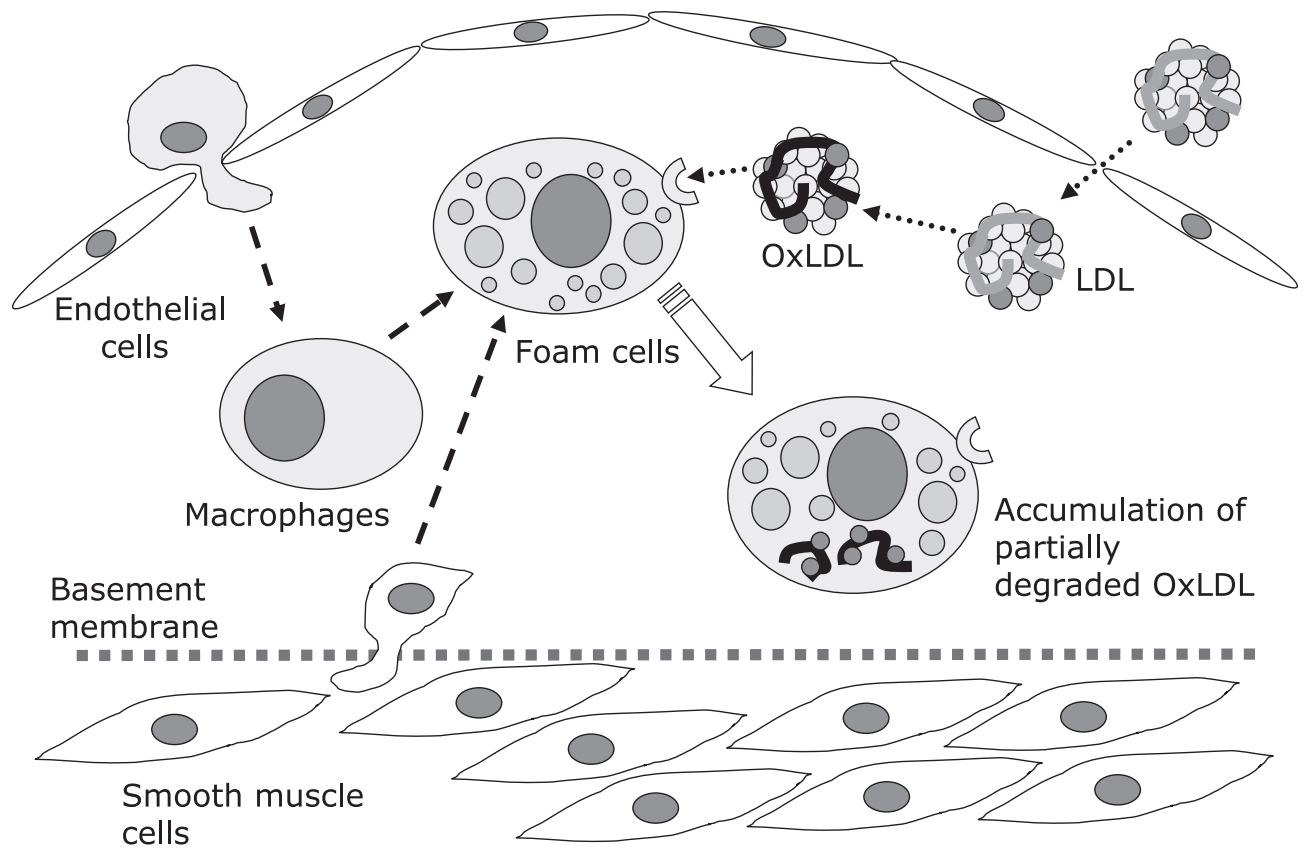

Fig. 2. Foam cell formation and metabolism of OxLDL in atherosclerotic lesions. LDL particles are transferred into the vessel wall tissues, where they are oxidized. After the oxidation of LDL, the resulting OxLDL is recongnized by scavenger receptors and taken up by macrophages. A portion of the intimal smooth muscle cells de-differentiate and migrate into the area of intimal thickening. Activated smooth muscle cells are also able to accumulate OxLDL. OxLDL is degraded in lysosomes after being taken up by the cells, but this degradation is not fully completed. Partially degraded modified apoB fragments thus accumulate in the lesions.

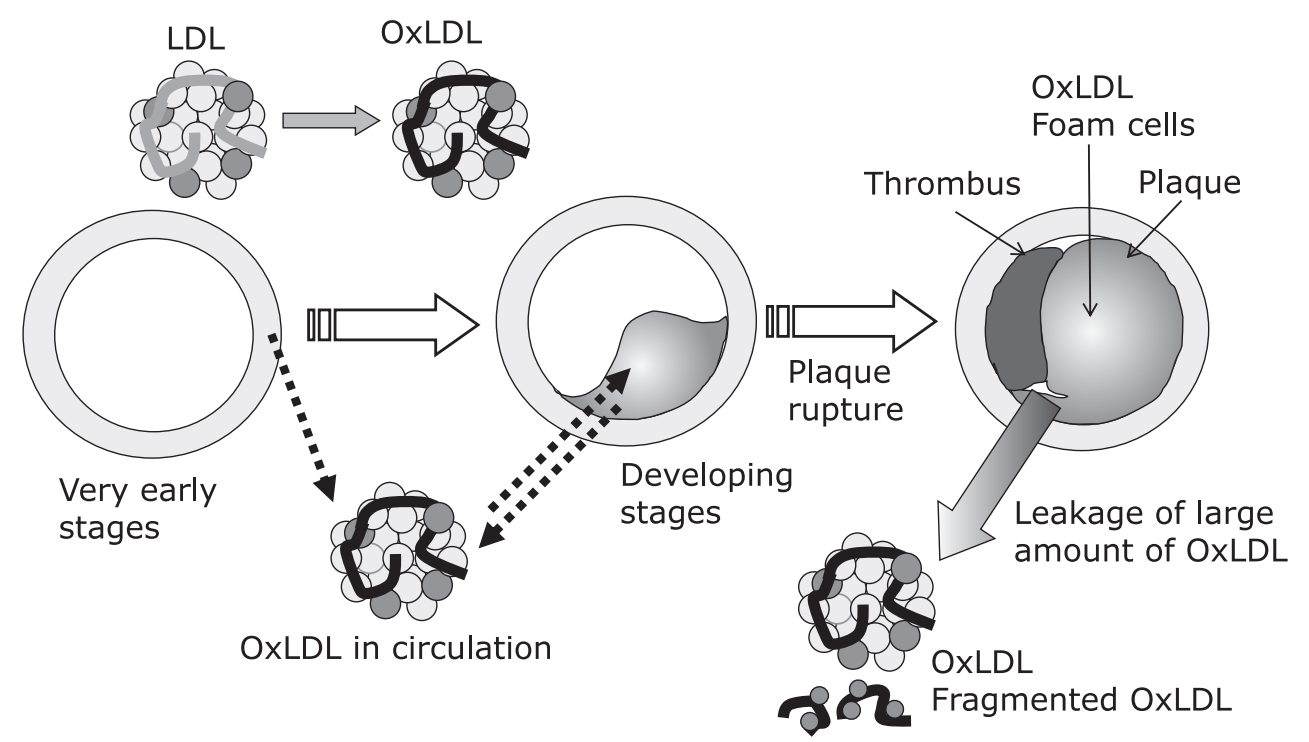

Fig. 3. Systemic behavior of OxLDL. OxLDL is thought to be generated in vessel wall tissues even in the early phase of atherogenesis. OxLDL gradally accumulates in premature lesions, while OxLDL migrates between the vessel wall and the circulation. When plaque is damaged, a thrombus is generated and the blood flow is occluded. In the acute phase upon plaque ruptures, a portion of the intact OxLDL and degraded OxLDL which has accumulated in the lesions is released into the circulation. In the peripheral blood, the OxLDL level go up in the acute phase, and it then goes down as OxLDL is cleared by the liver from the circulation.

levels using the mAb EO6. They have also reported increased OxLDL levels in patients with AMI. The OxLDL levels increased in the acute phase or after percutaneous intervention and transplantation, ${ }^{(21,22)}$ suggesting that plaque injury after spontaneous rupture or clinical treatment would release OxLDL from the atherosclerotic lesions into the circulation. These observations taken together support the concept that OxLDL is released from ruptured lesions into the circulation.

A commercial kit to measure OxLDL is available from the Mercodia AB (Uppsala, Sweden). A number of studies were carried out using it, and an increase in the plasma OxLDL concentration in the patients with plaque instability was demonstrated. ${ }^{(23)}$ 

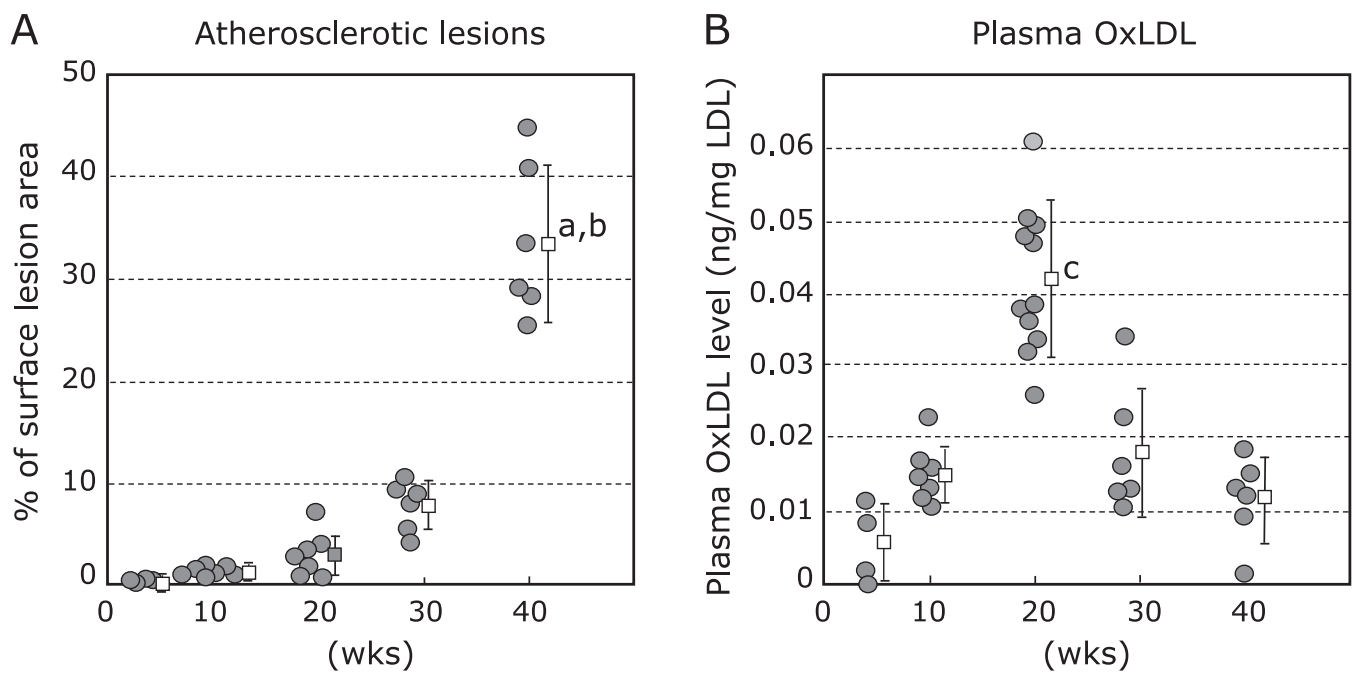

Fig. 4. The age-dependent changes in the atherosclerotic lesions and plasma OxLDL levels in apoE-KO mice. Male apoE-KO mice were maintained on a chow diet for up to 40 weeks. A: Whole aortas were stained with oil-red $\mathrm{O}$ and the percentage lesion area in the aortic surface was calculated. B: The LDL fraction was separated from each mouse plasma sample. OxLDL levels in the mouse LDL fractions were measured using the anti-OxPC mAb DLH3. Data for each mouse (circle) and the mean \pm standard deviation (square) are indicated. $a, p<0.01$ from the value at 4 weeks. $b, p<0.05$ from the values at 10 and 20 weeks. $c, p<0.01$ from any other values determined. (Cited from ref. 28 in a modified form with permission by the publisher.)

This kit is based on a competitive ELISA using a single antimodified apoB $\mathrm{mAb}$. It should be noted, however, that their method has quite different features from ours in two respects. First, their $\mathrm{mAb}$ binds to a hidden peptide fragment of the apoB protein which is stretched out along the OxLDL surface by a conformational change of apoB. ${ }^{(24)}$ Strictly speaking, this epitope is exposed to the surface of the LDL particles either by oxidation or other modification of apoB. Second, the assay procedure is a competitive ELISA using only one mAb, suggesting that the specificity of the assay may not be sufficiently high to detect very small amounts of OxLDL in the circulation. Some research groups have pointed out that this ELISA kit failed to detect differences between AMI patients and healthy subjects. ${ }^{(25)}$

\section{Behavior of OxLDL during Atherogenesis}

It has been an ongoing debate for many years whether OxLDL is the cause of atherosclerosis or the result of plaque formation. It is clear from a number of in vitro studies that OxLDL induces inflammatory reactions by activating many types of cells in the vascular wall, including macrophages, smooth muscle cells and endothelial cells, suggesting these reactions are a strong factor in the promotion of atherosclerotic lesion formation. And it is also very likely that inflammation responses increase reactive oxygen species (ROS) production and may thus initiate oxidation reactions of PUFA.

We thought it is important to study the early events in the vessel walls which take place in the course of atherogenesis to answer this question. It is reported that early lesions in human coronary arteries appear in subjects as young as 20 years old, ${ }^{(26)}$ but it takes decades to develop large and severe lesions, and cardiovascular events start to occur in the decades of the age of 50's or 60's. Since it is not easy to study the very early steps of atherogenesis in humans, we examined apoE-knockout (apoE-KO) mice. ApoE$\mathrm{KO}$ mice have been used worldwide as a model animal of spontaneous hyperlipidemia and atherosclerosis. ${ }^{(27)}$

ApoE-KO mice were maintained on a normal chow for up to 40 weeks. Very small atherosclerotic lesions appeared as early as 10 weeks, and became extensively enlarged after 20 weeks of age. The plasma OxLDL level in the apoE-KO mice increased temporarily at 20 weeks and then decreased to the basal level. This increase in plasma OxLDL level was observed before the expansion of the lesion area (Fig. 4).(28) This observation suggests that LDL is oxidized even in the absence of atherosclerotic lesions, and that OxLDL may be involved in the subsequent development of the lesions. We detected two oxidation epitopes, $\mathrm{OxPC}$ and acrolein, in the aortic intima of the apoE-KO mice as early as 4 week of age.

One interesting question is why the plasma OxLDL level decreases after the temporal rise at 20 weeks of age. Since the OxLDL appeared to accumulate in the aortic lesions during the expansion of the lesions, it seems highly likely that OxLDL is transferred from the circulation into the vessel wall tissues. Another question is why OxLDL increases in the plasma, since it is thought that LDL is oxidized in the vessel wall tissues and the serum itself has a strong antioxidant capacity. It is possible that OxLDL may easily move between the circulation and vessel wall tissues. Witztum and colleagues demonstrated that the OxLDL levels in the plasma and in lesions change during the progression and regression of aortic lesions in cynomolgus monkeys. ${ }^{(29)}$ Their observations also strongly suggest there is active transport of OxLDL between the circulation and vessel wall tissues.

\section{The Presence of OxPC in Pathological Lesions}

Age-related macular degeneration (AMD) is characterized by a progressive degeneration of the neurosensory retina, retinal pigment epithelium and choriocapillaris in the macular area. The number of patients increasing and AMD has become a leading cause of blindness in the elderly population in Japan. Kamei et al. ${ }^{(30)}$ found a strong positivity to the $\mathrm{mAb} \mathrm{DLH} 3$ in retinas from patients with AMD. OxPC was much less commonly found in the healthy retina even though the donors were older. The presence of OxPC-modified proteins in the retina was also confirmed by Western blotting.

An accumulation of OxPC was found in the chondrocytes in cartilage from patients with osteoarthritis. ${ }^{(31)}$ Osteoarthritis is a local inflammatory disease in joint synovial membranes. Cartilage is a unique tissue in which few cells are scattered in a very thick layer of extracellular matrix and there are very few vessels. 


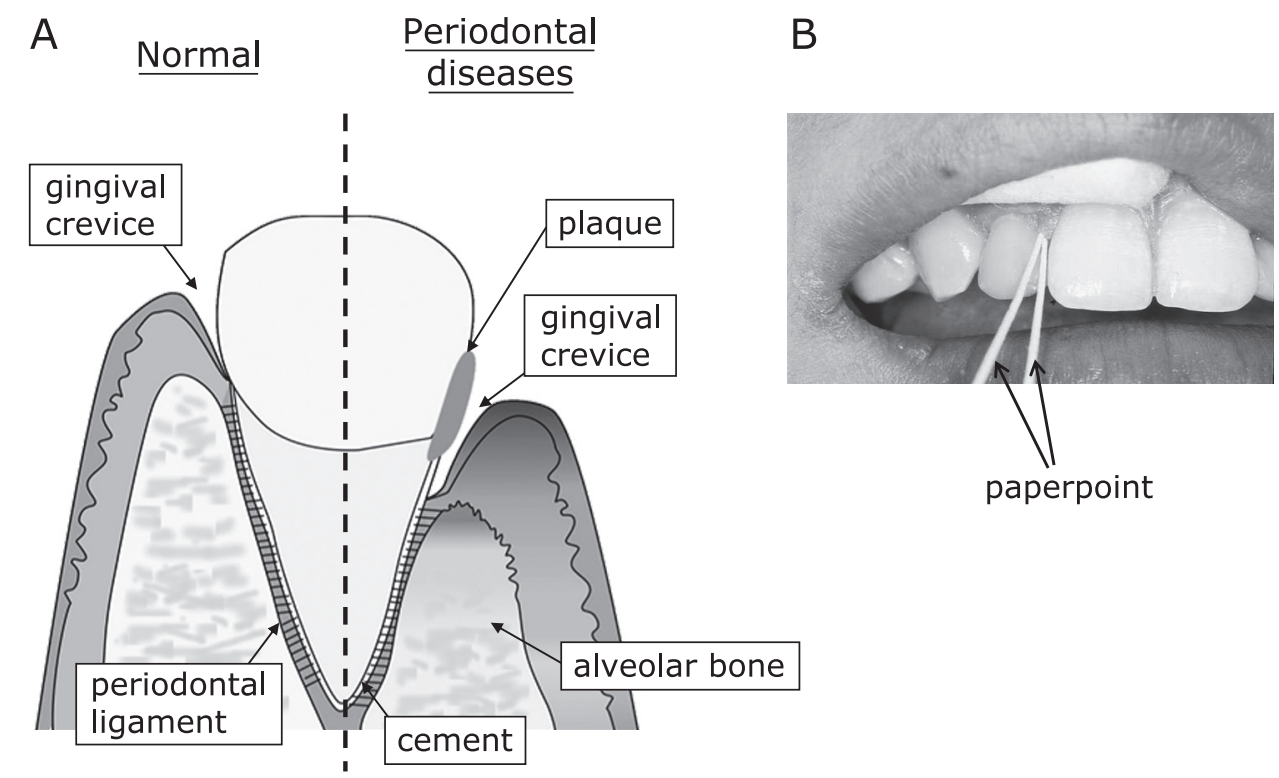

Fig. 5. A: An outline of the gingival tissues is illustrated. A tooth is mechanically supported by alveolar bone, and the tooth and the alveolar bone are surrounded by gingiva. The tooth and the alveolar bone are tightly attached by the thin gingival membrane called periodontal ligament. On the top of the periodontal ligament, there is gingival crevice which is a small space between the tooth and gingival epithelia. Gingival crevicular fluid (GCF) is an exudate recovered from the crevice. When the tissue is injured from periodontitis, the alveolar bone and the periodontal ligament are reduced and the gingival crevice deepens. B: GCF is easily and safely recovered using a needle-shaped filter paper called a paperpoint.

When a peripheral nerve fiber is injured, the axon and myelin sheath are regenerated. In this process many types of cells are involved, including Schwann cells, macrophages and fibroblasts are involved. Macrophages are thought to have a scavenging role in which damaged cells and cell debris are recognized by scavenger receptors and cleared away by endocytosis. Oxidative modification of membranes could be involved in this cellular damage of nerve cells, since OxPC has been shown to accumulate in the sciatic nerve of CD36-knockoput mice after crush injury. ${ }^{(32)}$

Lipid peroxidation can proceed not only in lipoproteins but a lipid environment, such as cellular membranes. The presence of $\mathrm{OxPC}$ in certain disease lesions suggests that either oxidative damage of cell components has occurred or oxidized materials such as OxLDL have accumulated in local tissues. It is thus a reasonable hypothesis that cellular membranes in the retina, chondrocytes and nerve cells are oxidized under certain pathological conditions.

\section{The Presence of OxLDL in the Oral Environment}

In developed countries, the number of patients with periodontitis has been increasing, and now periodontitis is the primary cause of tooth loss. Evidence has accumulated for a close relationship between atherosclerosis and periodontal diseases. For example, epidemiological studies have shown a significantly higher probability of periodontitis in patients with hypercholesterolemia and cardiovascular disease. ${ }^{(33-35)}$ The presence of Porphyromonas gingivalis in atherosclerotic lesions, one of the major culprit bacterial species of periodontitis, has been demonstrated. ${ }^{(36)}$ When $P$. gingivalis was injected in apoE-KO mice, the aortic lesions increased significantly. ${ }^{(37,38)}$

The gingiva is made up of the tissues surrounding the teeth and alveolar bones. Since these tissues are exposed to air and/or food essentially all the time, it is a front-line region of bacterial infection in oral tissue. Teeth are fixed by the alveolar bones together with the support of the periodontal ligament, which is attached to the gingival tissues (Fig. 5). Gingival crevicular fluid $(\mathrm{GCF})$ is an exudate from gingival tissues that originates from plasma, so that many of the plasma proteins are detected in GCF in addition to certain gingival tissue proteins which are secreted. ${ }^{(39)}$ For example, $\alpha$-defensin, a potent anti-inflammatory peptide, has reportedly been found in GCF. ${ }^{(40)}$ However, the information on GCF thus far is very limited.

One interesting feature of GCF is that it can be easily and safely recovered from the gingival crevice, suggesting that it is potentially useful as a clinical target of biochemical evaluation. We collected GCF at the sites of healthy teeth from patients with dental problems but no evident systemic or other infection. The presence of apoB was demonstrated by Western blotting and the presence of OxLDL was observed using sandwich ELISA. ${ }^{(41)}$ Interestingly, the OxLDL level in GCF from healthy subjects was 17 times higher than the plasma OxLDL levels in the same subjects on average, suggesting that LDL is oxidized in local gingival tissues (Fig. 6). The GCF OxLDL levels relatively large deviations, it did not reach significant difference. The GCF OxLDL levels might, therefore, be a useful marker of the oxidative status in our body. Since the relationship between periodontitis and metabolic disorders such as diabetic mellitus and atherosclerosis has come to be a focus of attention recently, ${ }^{(33-38)}$ it is important to study the relative OxLDL levels in patients with these metabolic disorders so as to eventually obtain simple and effective clinical tests.

\section{OxLDL as an Inflammatory Stimulant in Oral Disease}

A number of studies have shown that OxLDL is a potent stimulant of various types of activity in vessel wall cells, for example, the proliferation of endothelial cells, smooth muscle cells and macrophages, ${ }^{(42,43)}$ the production of cytokines and other mediators, ${ }^{(44-46)}$ platelet activation ${ }^{(47)}$ and the induction of cell death. ${ }^{(48,49)}$

However, the effects of OxLDL on other types of cells, such as epithelial cells, have not been well studied yet. There is a paper reporting the effects of OxLDL on NRK52E proximal tubule epithelial cells, in which the up-regulation of ICAM-1 and focal adhesion kinase was observed. ${ }^{(50)}$

We thought that the OxLDL identified in GCF might be actively 


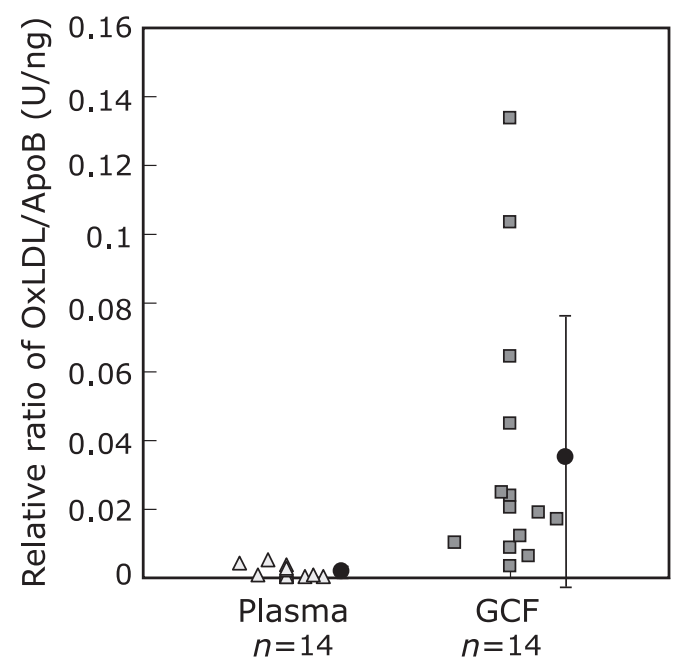

Fig. 6. The OxLDL/LDL ratios in GCF and plasma. GCF (square) and plasma samples (triangle) were collected from the 14 healthy subjects, and the concentrations of OxLDL and LDL were determined by ELISA to obtain OxLDL/LDL ratios. The mean \pm standard deviation was indicated with closed circle. It is noted that the OxLDL/LDL ratio in GCF was 17 times higher than that in plasma on average. (Cited from ref. 41 in a modified form under permission from the publisher.)

involved in the inflammatory responses in oral tissues. The oral epithelial cell line Ca9-22 was incubated with OxLDL and the effect on the production of inflammatory factors was examined. Ca9-22 exhibited increased amounts of interleukin-8 (IL-8), prostaglandin $\mathrm{E}_{2}\left(\mathrm{PGE}_{2}\right)$ and IL-1 $\beta$ when stimulated with OxLDL. ${ }^{(51)}$ Treatment of the cells with lipopolysaccharide from $P$. gingivalis did not induce any change in the production of these inflammatory mediators, suggesting that OxLDL itself, rather than bacterial infection, stimulates oral epithelial cells. Oral keratinocytes, primary culture cells originating from human oral tissues, were also responsive to OxLDL and displayed increased production of IL-8, PGE 2 and IL- $1 \beta$ in the presence of OxLDL. ${ }^{(51)}$

The cellular signaling mechanisms for the OxLDL-dependent responses have been extensively studied using macrophages and endothelial cells. There are many receptor candidates for the mediation of the cell stimulation by OxLDL. Scavenger receptors bind OxLDL and take it up into cells. Among the reported scavenger receptors, $\mathrm{CD} 36$ and LOX-1 are thought to mediate cellular signaling as well as OxLDL uptake. ${ }^{(52-54)}$ In addition to the scavenger receptors, toll-like receptor 4 (TLR4), a receptor for bacterial endotoxin lipid A, also binds to OxLDL and is involved in OxLDL signaling. ${ }^{(55)}$ In the interior of cells, there also are multiple pathways activated by OxLDL, such as the MAP-kinase pathway and peroxisome proliferator-activated receptor $\gamma$ (PPAR $\gamma){ }^{(56-58)}$ Recently, another signaling molecule TLR4 that is activated in a variety of cells by OxLDL has come to be a focus of attention. ${ }^{(59-61)}$

It is evidently a very complex situation in which the responsible receptors and signaling mechanisms are not only multiple they also depend on the cell type. The effects of OxLDL on Ca9-22 cells were attenuated by fucoidan or dextransulfate, which are negatively charged polysaccharides, which suggests that there are scavenger receptors involved in the response. ${ }^{(62)} \mathrm{Ca} 9-22$ cells express the mRNA of the major scavenger receptors, including
CD36 and LOX-1, but we could not detect TLR4 mRNA (unpublished data). More work is needed to clarify the responsible receptor(s) for the OxLDL-dependent stimulation which takes place in oral epithelial cells. We also found that the nuclear factorkappa B (NF- $\mathrm{B}$ ) pathway is involved in the OxLDL-dependent production of IL- 8 in Ca9-22 cells and human oral keratino-

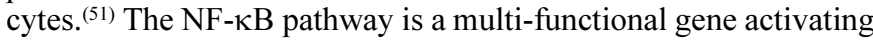
system, and it is well known that oxidative stress such as ROS activate the NF- $\kappa$ B pathway in a great variety of cells.

\section{Conclusion}

These results, taken together with those reported by others, suggest that OxLDL is a pro-inflammatory stimulant, and furthermore, that it is involved in not only atherosclerosis, but also other types of diseases, including periodontitis. Since OxLDL is present not only in local sites but also the systemic circulation local sites in various tissues, it has the capacity to induce inflammation in a variety of tissues, such as the vessel wall, retina, and gingiva. The systemic OxLDL level in the body may readily lend itself to the development of tools which will enable an early diagnosis and faster initiation of treatment.

\section{Conflict of Interest}

No potential conflicts of interest were disclosed.

\section{Acknowledgments}

I thank Dr. Tatsuya Takano (Teikyo University Faulty of Pharmaceutical Sciences) for the suggestion of the link to atherosclerosis research. I thank all the collaborators, especially Dr. Makiko Ueda (Osaka City University School of Medicine) for the studies on cardiovascular diseases, Dr. Masaaki Uno (Tokushima University School of Medicine) for the studies on carotid arteriosclerosis, and Dr. Matsuo Yamamoto (Showa University Dental Hospital) for the studies on collaboration in periodontal studies. I also thank Drs. Rina Kato and Takashi Obama (Showa University School of Pharmacy) for an extensive contribution in driving forward a series of our research. Pacific Edit reviewed the manuscript prior to submission.

\section{Abbreviations}

AMD age-related macular degeneration

AMI acute myocardial infarction

apoB apolipoprotein B

apoE apolipoprotein $\mathrm{E}$

apoE-KO apolipoprotein E-knockout

ELISA enzyme-linked immunosorbent assay

GCF gingival crevicular fluid

IL interleukin

LDL low-density lipoprotein

$\mathrm{mAb}$ monoclonal antibody

$\mathrm{NF}-\kappa \mathrm{B}$ nuclear factor-kappa B

OxLDL oxidatively modified low-density lipoprotein

OxPC oxidized phosphatidylcholine

$\mathrm{pAb} \quad$ polyclonal antibody

PC phosphatidylcholine

$\mathrm{PGE}_{2} \quad$ prostaglandin $\mathrm{E}_{2}$

PUFA polyunsaturated fatty acids

ROS reactive oxygen species

TLR4 Toll-like receptor 4

\section{References}

1 Stocker R. Lipoprotein oxidation: mechanistic aspects, methodological approaches and clinical relevenace. Curr Opin Lipidol 1994; 5: 422-433.

2 Steinbrecher UP, Parthasarathy S, Leake DS, Witztum JL, Steinberg D. Modification of low density lipoprotein by endothelial cells involves lipid 
peroxidation and degradation of low density lipoprotein phospholipids. Proc Natl Acad Sci USA 1984; 81: 3883-3887.

3 Kodama T, Freeman M, Rohrer L, Zabrecky J, Matsudaira P, Krieger M. Type I macrophage scavenger receptor contains alpha-helical and collagenlike coiled coils. Nature 1990; 343: 531-535.

4 Greaves DR, Gordon S. Thematic review series: the immune system and atherogenesis. Recent insights into the biology of macrophage scavenger receptors. J Lipid Res 2005; 46: 11-20.

5 Kimura J, Nakagami K, Amanuma K, Ohkuma S, Yoshida Y, Takano T. Monoclonal antibodies recognizing lipid-laden cells and extracellular regions with lipid-deposits in atherosclerotic aorta. Virchows Arch A Pathol Anat Histopathol 1986; 410: 159-164.

6 Mori M, Itabe H, Takatoku K, et al. Presence of phospholipid-neutral lipid complex structures in atherosclerotic lesions as detected by a novel monoclonal antibody. J Biol Chem 1999; 274: 24828-24837.

7 Itabe H, Jimi S, Kamimura S, et al. Appearance of cross linked proteins in human atheroma and rat pre-fibrotic liver detected by a new monoclonal antibody. Biochim Biophys Acta 1998; 1406: 28-39.

8 Itabe $\mathrm{H}$, Takeshima $\mathrm{E}$, Iwasaki $\mathrm{H}$, et al. A monoclonal antibody against oxidized lipoprotein recognizes foam cells in atherosclerotic lesions. Complex formation of oxidized phosphatidylcholine and polypeptides. J Biol Chem 1994; 269: 15274-15279.

9 Itabe H, Yamamoto H, Imanaka T, et al. Oxidized phosphatidylcholines that modify proteins. Analysis by monoclonal antibody against oxidized low density lipoprotein. J Biol Chem 1996; 271: 33208-33217.

10 Spiteller G. Peroxyl radicals: inductors of neurodegenerative and other inflammatory diseases. Their origin and how they transform cholesterol, phospholipids, plasmalogens, polyunsaturated fatty acids, sugars, and proteins into deleterious products. Free Radic Biol Med 2006; 41: 362-387.

11 Ehara S, Ueda M, Naruko T, et al. Elevated levels of oxidized low density lipoprotein show a positive relationship with the severity of acute coronary syndromes. Circulation 2001; 103: 1955-1960.

12 Nishi K, Itabe H, Uno M, et al. Oxidized LDL in carotid plaques and plasma associates with plaque instability. Arterioscler Thromb Vasc Biol 2002; 22: 1649-1654.

13 Lougheed M, Moore ED, Scriven DR, Steinbrecher UP. Uptake of oxidized LDL by macrophages differs from that of acetyl LDL and leads to expansion of an acidic endolysosomal compartment. Arterioscler Thromb Vasc Biol 1999; 19: 1881-1890.

14 Itabe H, Suzuki K, Tsukamoto Y, et al. Lysosomal accumulation of oxidized phosphatidylcholine-apolipoprotein B complex in macrophages: intracellular fate of oxidized low density lipoprotein. Biochim Biophys Acta 2000; 1487: 233-245.

15 Itabe H, Yamamoto H, Imanaka T, et al. Sensitive detection of oxidatively modified low density lipoprotein using a monoclonal antibody. J Lipid Res 1996; 37: 45-53.

16 Uno M, Kitazato KT, Nishi K, Itabe H, Nagahiro S. Raised plasma oxidised LDL in acute cerebral infarction. J Neurol Neurosurg Psychiatry 2003; 74: 312-316.

17 Itabe H, Ueda M. Measurement of plasma oxidized low-density lipoprotein and its clinical implications. J Atheroscler Thromb 2007; 14: 1-11.

18 Naruko T, Ueda M, Ehara S, et al. Persistent high levels of plasma oxidized low-density lipoprotein after acute myocardial infarction predict stent restenosis. Arterioscler Thromb Vasc Biol 2006; 26: 877-883.

19 Itabe H. Oxidized low density lipoprotein: what is understood and what remains to be clarified. Biol Pharm Bull 2003; 26: 1-9.

20 Tsimikas S, Bergmark C, Beyer RW, et al. Temporal increases in plasma markers of oxidized low-density lipoprotein strongly reflect the presence of acute coronary syndromes. J Am Coll Cardiol 2003; 41: 360-370.

21 Tsimikas S, Lau HK, Han KR, et al. Percutaneous coronary intervention results in acute increases in oxidized phospholipids and lipoprotein(a): shortterm and long-term immunologic responses to oxidized low-density lipoprotein. Circulation 2004; 109: 3164-3170.

22 Fang JC, Kinlay S, Behrendt D, et al. Circulating autoantibodies to oxidized LDL correlate with impaired coronary endothelial function after cardiac transplantation. Arterioscler Thromb Vasc Biol 2002; 22: 2044-2048.

23 Holvoet P, Van Cleemput J, Collen D, Vanhaecke J. Oxidized low density lipoprotein is a prognostic marker of transplant-associated coronary artery disease. Arterioscler Thromb Vasc Biol 2000; 20: 698-702.

24 Holvoet P, Donck J, Landeloos M, et al. Correlation between oxidized low density lipoproteins and von Willebrand factor chronic renal failure. Thromb Haemost 1996; 76: 663-669.
25 Braun S, Ndrepepa G, von Beckerath N, et al. Lack of association between circulating levels of plasma oxidized low-density lipoproteins and clinical outcome after coronary stenting. Am Heart J 2005; 150: 550-556.

26 Velican C, Velican D. The precursors of coronary atherosclerotic plaques in subjects up to 40 years old. Atherosclerosis 1980; 37: 33-46.

27 Meir KS, Leitersdorf E. Atherosclerosis in the apolipoprotein-E-deficient mouse: a decade of progress. Arterioscler Thromb Vasc Biol 2004; 24: 10061014.

28 Kato R, Mori C, Kitazato K, et al. Transient increase in plasma oxidized LDL during the progression of atherosclerosis in apolipoprotein $\mathrm{E}$ knockout mice. Arterioscler Thromb Vasc Biol 2009; 29: 33-39.

29 Tsimikas S, Aikawa M, Miller FJ Jr., et al. Increased plasma oxidized phospholipids: apolipoprotein B-100 ratio with concomitant depletion of oxidized phospholipids from atherosclerotic lesions after dietary lipid-lowering: a potential biomarker of early atherosclerosis regression. Arterioscler Thromb Vasc Biol 2007; 27: 175-181.

30 Suzuki M, Kamei M, Itabe H, et al. Oxidized phospholipids in the macula increase with age and in eyes with age-related macular degeneration. Mol Vis 2007; 23: 772-778.

31 Akagi M, Kanata S, Mori S, Itabe H, Sawamura T, Hamanishi C. Possible involvement of the oxidized low-density lipoprotein/lectin-like oxidized lowdensity lipoprotein receptor-1 system in pathogenesis and progression of human osteoarthritis. Osteoarthritis Cartilage 2007; 15: 281-290.

32 Eto M, Yoshikawa H, Fujimura H, et al. The role of CD36 in peripheral nerve remyelination after crush injury. Eur J Neurosci 2003; 17: 2659-2666.

33 Dietrich T, Jimenez M, Krall Kaye EA, Vokonas PS, Garcia RI. Agedependent associations between chronic periodontitis/edentulism and risk of coronary heart disease. Circulation 2008; 117: 1668-1674.

34 Tonetti MS, D'Aiuto F, Nibali L, et al. Treatment of periodontitis and endothelial function. $N$ Engl J Med 2007; 356: 911-920.

35 Bahekar AA, Singh S, Saha S, Molnar J, Arora R. The prevalence and incidence of coronary heart disease is significantly increased in periodontitis: a meta-analysis. Am Heart J 2007; 154: 830-837.

36 Haraszthy VI, Zambon JJ, Trevisan M, Zeid M, Genco RJ. Identification of periodontal pathogens in atheromatous plaques. J Periodontol 2000; 71: 1554-1560.

37 Li L, Messas E, Batista EL Jr., Levine RA, Amar S. Porphyromonas gingivalis infection accelerates the progression of atherosclerosis in a heterozygous apolipoprotein E-deficient murine model. Circulation 2002; 105: 861-867.

38 Lalla E, Lamster IB, Hofmann MA, et al. Oral infection with a periodontal pathogen accelerates early atherosclerosis in apolipoprotein E-null mice. Arterioscler Thromb Vasc Biol 2003; 23: 1405-1411.

39 Delima AJ, van Dyke TE. Origin and function of the cellular components in gingival crevice fluid. Periodontol 2000 2003; 31: 55-76.

40 Pisano E, Cabras T, Montaldo C, et al. Peptides of human gingival crevicular fluid determined by HPLC-ESI-MS. Eur J Oral Sci 2005; 113: 462-468.

41 Sakiyama Y, Kato R, Inoue S, Suzuki K, Itabe H, Yamamoto M. Detection of oxidized low-density lipoproteins in gingival crevicular fluid from dental patients. J Periodontal Res 2010; 45: 216-222.

42 Augé N, Andriew N, Nègre-Salvayre A, Thiers JC, Levade T, Salvayre R. The sphingomyelin-ceramide signaling pathway is involved in oxidized low density lipoprotein-induces cell proliferation. J Biol Chem 1996; 271: 19251-19255.

43 Kusuhara M, Chait A, Cader A, Berk BC. Oxidized LDL stimulates mitogenactivated protein kinases in smooth muscle cells and macrophages. Arterioscler Thromb Vasc Biol 1997; 17: 141-148.

44 Hamilton TA, Major JA, Chisolm GM. The effects of oxidized low density lipoproteins on inducible mouse macrophage gene expression are gene and stimulus dependent. J Clin Invest 1995; 95: 2020-2027.

45 Lehr HA, Hübner C, Finckh B, et al. Role of leukotriens in leukocyte adhesion following systemic administration of oxidatively modified human low density lipoprotein in hamsters. J Clin Invest 1991; 88: 9-14.

46 Higashi Y, Peng T, Du J, et al. A redox-sensitive pathway mediates oxidized LDL-induced downregulation of insulin-like growth factor-1 receptor. J Lipid Res 2005; 46: 1266-1277.

47 Ishii H, Kizaki K, Horie S, Kazama M. Oxidized low density lipoprotein reduces thrombomodulin transcription in cultured human endothelial cells through degradation of the lipoprotein in lysosomes. J Biol Chem 1996; 271: 8458-8465.

48 Sata M, Walsh K. Endothelial cell apoptosis induced by oxidized LDL is associated with the down-regulation of the cellular caspase inhibitor FLIP. $J$ Biol Chem 1998; 273: 33103-33106. 
49 Sanson M, Augé N, Vindis C, et al. Oxidized low-density lipoproteins trigger endoplasmic reticulum stress in vascular cells: prevention by oxygen-regulated protein 150 expression. Circ Res 2009; 104: 328-336.

50 Kelly KJ, Wu P, Patterson CE, Temm C, Dominguez JH. LOX-1 and inflammation: a new mechanism for renal injury in obesity and diabetes. $\mathrm{Am} \mathrm{J}$ Physiol Renal Physiol 2008; 294: F1136-F1145.

51 Nagahama Y, Obama T, Usui M, et al. Oxidized low-density lipoproteininduced periodontal inflammation is associated with the up-regulation of cyclooxygenase- 2 and microsomal prostaglandin synthase 1 in human gingival epithelial cells. Biochem Biophys Res Commun 2011; 413: 566-571.

52 Park YM, Febbraio M, Silverstein RL. CD36 modulates migration of mouse and human macrophages in response to oxidized LDL and may contribute to macrophage trapping in the arterial intima. J Clin Invest 2009; 119: 136-145.

$53 \mathrm{Li} \mathrm{D}$, Mehta JL. Antisense to LOX-1 inhibits oxidized LDL-mediated upregulation of monocyte chemoattractant protein-1 and monocyte adhesion to human coronary artery endothelial cells. Circulation 2000; 101: 28892895.

54 Tanigawa H, Miura S, Matsuo Y, Fujino M, Sawamura T, Saku K. Dominant-negative Lox-1 blocks homodimerization of wild-type Lox-1induced cell proliferation through exracellular signal regulated kinase $1 / 2$ activation. Hypertension 2006; 48: 294-300.

55 Miller YI, Viriyakosol S, Worrall DS, Boullier A, Butler S, Witztum JL. Toll-like receptor 4-dependent and -independent cytokine secretion induced by minimally oxidized low-density lipoprotein in macrophages. Arterioscler Thromb Vasc Biol 2005; 25: 1213-1219.
56 Taketa K, Matsumura T, Yano M, et al. Oxidized low density lipoprotein activates peroxisome proliferator-activated recepter-alpha (PPARalpha) and PPARgamma through MAPK-dependent COX-2 expression in macrophages. J Biol Chem 2008; 283: 9852-9862.

57 Nagy L, Tontonoz P, Alvarez JG, Chen H, Evans RM. Oxidized LDL regulates macrophage gene expression through ligand activation of PPAR $\gamma$. Cell 1998; 93: 229-240.

58 Inoue $\mathrm{M}$, Itoh $\mathrm{H}$, Tanak $\mathrm{T}$, et al. Oxidized LDL regulates vascular endothelial growth factor expression in human macrophages and endothelial cells through activation of peroxisome proliferator-activated receptor- $\gamma$. Arterioscler Thromb Vasc Biol 2001; 21: 560-566.

59 Wiesner P, Choi SH, Almazan F, et al. Low dose of lipopolysaccharide and minimally oxidized low-density lipoprotein cooperatively activate macrophages via nuclear factor $\kappa \mathrm{B}$ and activator protein-1: possible mechanism for acceleration of atherosclerosis by subclinical endotoxemia. Circ Res 2010; 107: 56-65.

60 Stewart CR, Stuart LM, Wilkinson K, et al. CD36 ligands promote sterile inflammation through assembly of a Toll-like receptor 4 and 6 heterodimer. Nat Immunol 2010; 11: 155-161.

61 Wu CC, Wang SH, Kuan II, et al. OxLDL upregulates caveolin-1 expression in macrophages: role for caveolin-1 in the adhesion of oxLDL-treated macrophages to endothelium. J Cell Biochem 2009; 107: 460-472.

62 Suzuki K, Sakiyama Y, Usui M, et al. Oxidized low-density lipoproteins increases IL-8 production in human gingival epithelial cell line Ca9-22. $J$ Periodontal Res 2010; 45: 488-495. 